\title{
Correction to: Functions in Python
}

\section{Correction to:}

\section{Chapter 11 in: J. Hunt,}

A Beginners Guide to Python 3 Programming, Undergraduate Topics in Computer Science, https://doi.org/10.1007/978-3-030-20290-3_11

In the original version of the book, the text "Rugby" has been replaced with "Python" in Chapter 11 page 126. The corrections have been carried out in the chapter. The erratum chapter and the book have been updated with the change. 